\title{
Special issue on intelligent analytics and management of mobile data and social media
}

\author{
Wen-Yang Lin ${ }^{1}$ • Hsin-Chang Yang ${ }^{2}$ • Tzung-Pei Hong ${ }^{1,3}$ • Leon S. L. Wang ${ }^{2}$
}

Published online: 11 July 2017

(C) Springer-Verlag GmbH Germany 2017

In the past decade, we have witnessed the explosive growth of mobile devices, including notebooks, smartphones, sensors, tablets and wearable devices, and social media that allows the creation and exchange of user-generated content, such as YouTube, Facebook, Twitter and Flickr. Now we are experiencing the blending of these two technologies, forming a widely adopted platform that reshapes nearly all aspects of our daily life, e.g., the way we connect people, doing business, entertaining, etc. This inspires enterprises to seek new business models and provides them greater opportunities than ever before to understand their customers, competitors, marketing trend by analyzing and managing data collected or created during the usage of mobile services and social media. However, specific characteristics of these data and media such as high variance in quality and usability, large volume, high velocity and low permanence make most contemporary data analysis and managing tools awkward to meet the enterprises' expectations.

Wen-Yang Lin

wylin@nuk.edu.tw

Hsin-Chang Yang

yanghc@nuk.edu.tw

Tzung-Pei Hong

tphong@nuk.edu.tw

Leon S. L. Wang

slwang@nuk.edu.tw

1 Department of Computer Science and Information Engineering, National University of Kaohsiung, Kaohsiung 811, Taiwan

2 Department of Information Management, National University of Kaohsiung, Kaohsiung, Taiwan

3 Department of Computer Science and Engineering, National Sun Yat-sen University, Kaohsiung, Taiwan
This special issue provides a showcase for recent research achievements and solutions in intelligent data analytics and management of mobile data and social media. Five papers are collected in this special issue, including two highly recommended papers from 12th International Conference on Advances in Mobile Computing and Multimedia (MoMM2014), each of which was reviewed by at least three reviewers to maintain high quality. In the following, we briefly introduce each paper.

The first paper Efficient Cluster-based Top-k Query Routing with Data Replication in MANETs by Amagata et al. (2015) considers the problem of improving query routing scheme in mobile ad hoc networks (MANETs). Based on a clustering framework for top- $k$ query processing, the proposed query routing protocol CTR retrieves only necessary data to reduce network traffic in MANETs. The authors also propose a query routing method $\mathrm{CTR}^{2}$ that integrates the clustering framework and a data replication approach. By retrieving only top- $k$ data from nearby nodes, $\mathrm{CTR}^{2}$ efficiently reduces the traffic and delay without sacrificing the accuracy of query results.

The second paper A Single-Directional Influence Topic Model Using Call and Proximity Logs Simultaneously by Han et al. (2015) reflects the new development in understanding social interactions through identifying interaction patterns sensed by mobile devices. Unlike most previous studies on this problem utilizing calls and proximity logs independently to represent social interactions, this paper considers calls and proximity logs simultaneously and proposes a single-directional influence topic model method. Experimental results show that the proposed method outperforms the counterpart that considers calls and proximities independently.

The third paper Link Prediction in Complex Network based on Modularity by Caiyan et al. (2016) presents a 
new approach for link prediction in complex networks. The problem of link prediction is to predict missing and potential links in a network. The proposed method solves the problem by first detecting communities in a network and then computing the similarity between nodes within the same community, with higher similarity reflecting stronger link. To this end, the authors introduced a new similarity measure, named modularity increment, which exploits the whole community structure information to evaluate the qualities of detected communities. Their approach was evaluated using six benchmark data sets and compared with eight traditional similarity-based link prediction methods. Experimental results show that the proposed method yields better quality.

The fourth paper $k^{-}$-Anonymization of Multiple Shortest Paths by Wang et al. (2016) is an interesting work on privacy preservation for social networks. Their method extends the well-known privacy model for anonymizing information network, called $k$-shortest path privacy, which minimally perturbing edge weights so that there are at least $k$ shortest paths between two vertices in the network. A main deficiency of $k$-shortest path privacy is that there may exist insufficient number of paths to be modified to meet the requirement. The proposed new model, named $k^{-}$-shortest path privacy, allows anonymizing node pairs with insufficient paths or edge weights. Empirical results conducted on two co-authorship networks demonstrate that the proposed method is more efficient and flexible than $k$-shortest path privacy.

The last paper DST: Days Spent Together Using Soft Sensory Information on OSNs-A Case Study on Facebook by Alzamzami et al. (2016) considered an interesting problem: How to measure the number of days spent together from face-to-face interactions on online social networks? By introducing the concept of soft sensory information on online social networks, i.e., the descriptive tags (time, location, people, etc.) added on shared multimedia, this paper proposes a method to estimate the number of days spent together by a given pair of users in a given duration. A preliminary experiment conducted on selected Facebook users show that the proposed method achieves about $70 \%$ accuracy.

We would like to express our sincere appreciation to all the authors for their contributions to this special issue. We also thank the referees for their careful reviews and valuable comments that assure the high quality of this issue. Our special thanks go to Professor Antonio Di Nola, the Editor-inChief, and Professor Vincenzo Loia, the Co-Editor-in-Chief, for their great support in publishing this special issue.

\section{Compliance with ethical standards}

Conflict of interest The authors declare that they have no conflict of interest.

\section{References}

Alzamzami F, Saini M, El Saddik A (2016) DST: days spent together using soft sensory information on OSNs-a case study on Facebook. Soft Comput. doi:10.1007/s00500-016-2175-1

Amagata D, Takahiro H, Yuya S, Shojiro N (2015) Efficient clusterbased top-k query routing with data replication in MANETs. Soft Comput. doi:10.1007/s00500-015-1867-2

Caiyan D, Chen L, Li B (2016) Link prediction in complex network based on modularity. Soft Comput. doi:10.1007/ s00500-016-2030-4

Han YJ, Park SY, Park SB (2015) A single-directional influence topic model using call and proximity logs simultaneously. Soft Comput. doi:10.1007/s00500-015-1898-8

Wang SL, Tsai YC, Hong TP, Kao HY (2016) $k^{-}$-anonymization of multiple shortest paths. Soft Comput. doi:10.1007/ s00500-016-2032-2 